\title{
Compatibility of Arbitrary Speeds with Special Relativity Axioms for Interior Dynamical Problems
}

\author{
Ruggero Maria Santilli \\ Thunder Energies Corporation, Tarpon Springs, Florida, U.S.A.
}

Email address:

research@i-b-r.org

To cite this article:

Ruggero Maria Santilli. Compatibility of Arbitrary Speeds with Special Relativity for Interior Dynamical Problems. American Journal of Modern Physics. Special Issue: Issue II: Foundations of Hadronic Mechanics. Vol. 5, No. 2-1, 2016, pp. 143-160.

doi: 10.11648/j.ajmp.2016050201.19

Received: September 2, 2015; Accepted: September 2, 2015; Published: June 1, 2016

Abstract: In this paper, we outline the rapidly growing literature on arbitrary speeds within physical media and show that, contrary to a widespread belief for one century, arbitrary speeds for interior dynamical problems are compatible with the abstract axioms of special relativity, provided that they are realized with the covering isomathematics specifically developed for the conditions considered. We finally point out a number of intriguing implications in cosmology, particle physics, nuclear physics, chemistry, gravitation, and mathematical models of interstellar travel.

Keyworks: Special Relativity, Superluminal Speeds, Isorelativity

\section{Maximal Speeds for Exterior Problems in Vacuum}

As it is well known, the advent of the Lorentz transformations [1]

$$
\begin{gathered}
x^{\prime 1}=x^{1}, x^{\prime 2}=x^{2}, \\
x^{\prime 3}=\gamma\left(x^{3}-\beta x^{4}\right), x^{\prime 4}=\gamma\left(x^{4}-\beta x^{3}\right), \\
\beta=\frac{v}{c}, \gamma=\frac{1}{\sqrt{1-\beta^{2}}} .
\end{gathered}
$$

and their extension by Poincaré [2] (hereon referred to as the Lorentz-Poincaré (LP) symmetry) leave invariant the line element in Minkowski space-time $M(x, \eta, I)$

$$
\begin{array}{r}
x^{2}=x_{1}^{2}+x_{2}^{2}+x_{3}^{2}-t^{2} c^{2}=x^{\mu} \eta_{\mu \nu} x^{v}, \\
x=\left(x^{\mu}\right)=\left(x_{1}^{\mu}-x_{2}^{\mu}\right), x^{4}=t, \mu, v=1,2,3,4, \\
\eta=\operatorname{Diag} \cdot\left(1,1,1,-c^{2}\right), I=\operatorname{Diag} \cdot(1,1,1,1),
\end{array}
$$

and are at the foundation of axioms of Special Relativity (SR) [3].

As it is also well known, symmetry (1) identifies the maximal causal speed for the conditions clearly expressed by Lorentz, Poincaré and Einstein [1-3] and experimentally confirmed, namely, for exterior dynamical problems, consisting of point particles and electromagnetic waves propagating in vacuum (conceived as empty space) when represented in an inertial reference frame.

In fact, the light cone, e.g., for infinitesimal displacements in $(3,4)$-dimensions

$$
\left(\delta x^{3}\right)^{2}-(\delta t)^{2} c^{2}=0,
$$

establishes the maximal causal speed in vacuum

$$
\frac{\delta x^{3}}{\delta t}=V_{\max }^{\text {vacuum }}=c .
$$

For decades, faster than light speeds (also called "superluminal speeds") were essentially ignored because they would violate causality and other physical laws. Nevertheless, with the passing of time the study of superluminal speeds became inevitable.

Nowadays, a search on superluminal speeds at the various archives in the internet shows the existence of a large number of papers published in refereed journals, thus suggesting a study on the problem of the causal and time invariant formulation of superluminal speeds.

Under a literature on superluminal speeds of such a dimension, we regret being unable to provide a comprehensive review, and are forced to quote a few representative illustrations of the studies considered in this 
paper, superluminal speeds of ordinary masses or electromagnetic waves, by deferring the study of tachyons (see, e.g., contributions by E. Recami and his group [47, 48]) to a separate paper.

\section{Superluminal Speeds in the Expansion of the Universe}

To our knowledge, studies of superluminal speeds were first motivated by the Doppler interpretation of the Hubble law [4] on the cosmological redshift of light

$$
z=\frac{\lambda_{o}}{\lambda_{e}}-1 \approx H d \equiv \frac{v}{c}
$$

where $\lambda_{o}\left(\lambda_{e}\right)$ is the wavelength of light at the origin (that observed on Earth), $d$ is the distance of a galaxy from Earth, and $H$ is the Hubble constant.

In fact, with the passing of the decades and the advances in telescopes, it became evident that the galaxies at the edge of the universe have values $z>1$ with consequential superluminal speeds. This occurrence can be assumed as signaling the initiationof studies in faster than light speeds. As an example, in 1966, Rees [6] attempted the reconciliation of superluminal galactic speeds with special relativity limit (4) by studying the possibility that superluminal speeds are illusory.

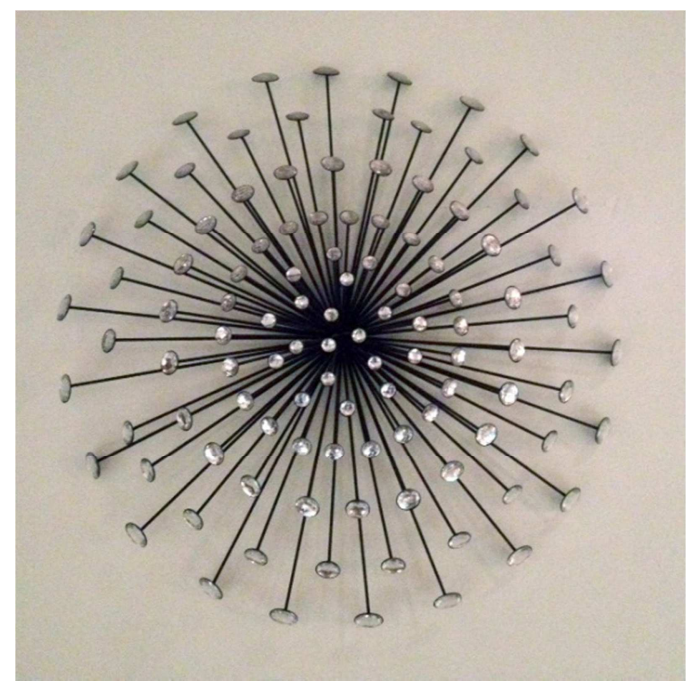

Figure 1. An artist rendering of the conjecture of the expansion of the universe (5) showing Earth necessarily at the center of the universe due to the dependence of the expansion speed $v=H c d$ on the distance $d$ in all "radial" directions from Earth. This return to the Middle Ages, which is inherent ion the conjecture of the expansion of the universe, is the historical reason for which Einstein, Hubble, Hoyle, Zwicky, de Broglie, Fermi and other famous scientists died without accepting the Doppler interpretation of the cosmological redshift [43-47]. Said interpretation became fashionable despite such authoritative oppositions because of the tacit intent of imposing the validity of the conventional interpretation of special relativity for the large scale structure of the universe.

It should be recalled that Einstein, Hubble, Hoyle, Zwicky, de Broglie, Fermi and other famous scientists died without accepting conjecture (5) on the Doppler interpretation of the cosmological redshift because the interpretation $H d=v / c$ holds in all possible radial directions from Earth, thus implying a necessary return to the Middle Ages with Earth at the center of the universe (Figure 1).

The same conclusion is inevitable for the conjecture of the big bang because, as a necessary condition to represent experimental data on the radial character of the cosmological redshift, the big bang must have occurred in our galactic vicinity, thus implying an "explosion" of the type depicted in Figure 1.

For the intent of avoiding Earth at the center of the universe, supporters of special relativity ventured the additional conjecture that space itself is expanding. However, it is known that this conjecture would have achieved its intent in the event the expansion of the universe were uniform. In reality, conjecture (5) intrinsically implies the acceleration of the expansion, that is, the increase of the speed of galaxies with the increase of their distance, $v=H c d$. This acceleration also occurs in all radial directions from Earth, thus implying again Earth at the center of the universe (see Figure 2 and Refs. [43-45]).

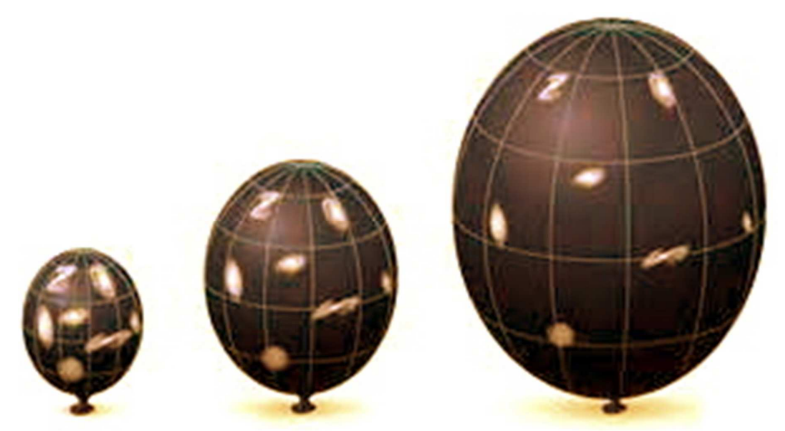

Figure 2. An illustration of the lack of the evidence that the conjecture of the expansion of space itself would provide a consistent representation of conjecture (5) in the event the expansion were uniform. However, conjecture (5) implies the increase of the speed $v=H c d$ in all radial directions from Earth that cannot possibly be consistent represented via the inflation of balloon [43-47].

In any case, it is easy to see that the sole geometry representing conjecture (5) is that with the shape of a funnel (Figure 3). However, a necessary condition to represent experimental data is that Earth is at the tip of the funnel evidently because all speeds $v$ are measured from Earth, thus implying again Earth at the center of the universe. Also, the funnel-type geometry causes a rather drastic departure from general relativity due to its irreconcilable incompatibility with the Riemannian geometry (Figure 3).

To confirm the sound vision by Einstein, Hubble, Hoyle, Zwicky, de Broglie, Fermi and other famous scientists, the implausibility of the expansion of the universe, is confirmed by the fact that the energy needed to accelerate billions and billions of galaxies is so disproportionately large to prevent any realistic model for its physical origin.

The yet additional conjecture that the universe is filled up with the mysterious and invisible dark energy was ventured to represent the expansion and inherent acceleration of the universe. However, no quantitative model has appeared to date 
in the refereed scientific literature achieving such a goal due to several technical insufficiencies, including the fact that, according to Albert Einstein, energy is the source of gravitational attraction, and certainly not of repulsion.

The above orthodox models are based on the conception of the large scale structure of the universe as an exterior dynamical problem consisting of particles and electromagnetic waves traveling in vacuum. This conception is evidently mandatory for the tacit intent of imposing the cosmological validity of the conventional interpretation of special relativity.

Following decades of cosmological studies, the author's conclusion is that the inconsistencies or sheers insufficiencies of the conjecture on the expansion of the universe are due to the fact that the cosmological redshift of galactic light characterizes a strictly interior dynamical problem consisting of particles and electromagnetic waves propagating within the intergalactic medium [9-16], mostly composed of hydrogen and other gases at absolute zero degree temperature, dust, cosmic rays, besides including light emitted by all stars in the universe.

As established in Refs. [43-47], the latter conception of the universe implies necessary, experimentally established deviations from the conventional interpretation of special relativity, with particular reference to deviations from the Doppler shift. However, as we shall see in this paper, the abstract axioms of special relativity remain valid provided that they are elaborated with a mathematics more appropriate for interior conditions.

The conception of the universe as an interior dynamical problem was pioneered in 1929 by Zwicky [5] who suggested the interpretation of the cosmological redshift $z=H d$ via the hypothesis that light loses energy during its long travel to reach Earth due to scattering with the intergalactic medium.

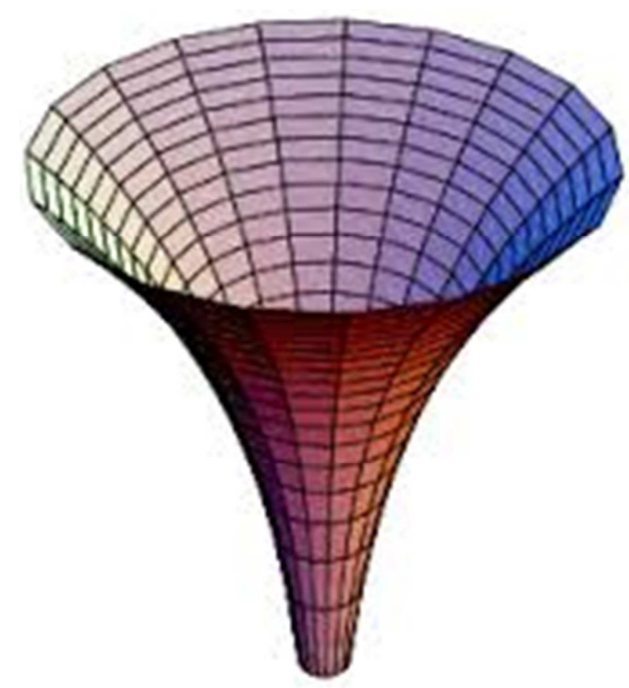

Figure 3. An illustration of the only known consistent representation of the expansion of the universe according to assumption (5) that represents the linear increase of the speed with the distance. However, a necessary condition for consistency is that Earth must be at the tip of the funnel, thus implying again Earth at the center of the universe. Additionally, the funnel geometry is irreconcilably incompatible with the Riemannian geometry of general relativity $[43=47]$.
Unfortunately for scientific knowledge, Zwicky's hypothesis was "disqualified" by the orthodox physics community, and remains "disqualified" to this day, because it clearly violates Einstein's special relativity, evidently due to the fact that, for Zwicky's hypothesis, light is no longer immutable as required by special relativity axioms.

However, Zwicky's hypothesis is experimentally verifiable on Earth, while all conjectures on the expansion of the universe are individually conceived not to be testable on Earth so that they can be imposed via abuses of academic credibility. The author has spent decades of research in the field and confirmed experimentally the validity of Zwicky's hypothesis with the consequential lack of the universe (see for brevity Refs. [43-45]).

The conception of cosmology as an interior dynamical problem is best illustrated by the redshift of galactic stars, which is anomalous in the sense that it is generally smaller (bigger) than the redshift of the galaxy as a whole for stars near (far away from) the galactic center.

Always for the intent of reconciling physical evidence with special relativity, the scientific community coordinated the conjecture that galaxies (as well as their clusters) are filled up with the mysterious, invisible and undetectable dark matter.

However, no quantitative model has been published in the refereed literature showing that dark matter achieves a quantitative representation of the anomalous galactic redshift (Figure 5). Besides, according to Newton, galaxies should contract in the event they are filled up with any type of matter, contrary to astrophysical evidence.

In papers $[46,47]$, the author has shown that the problem of the anomalous redshift of galactic stars is indeed a fully interior dynamical problem because the origin of the anomalies is entirely due to the loss (acquisition of energy of star light to (from) the actual material gas filling up all galaxies which is cold at the galactic periphery (very hot near its center).

The resulting frequency shift without any appreciable Doppler's contribution are today known as Santilli isoredshift (isoblueshift), where the prefix "iso" stands to denote their derivation via the coveting of 20th century mathematics known asisomathematics

Ref. [46,47] show in particular that, unlike the case for the conjecture of dark matter, the loss or acquisition of energy of star light from the innergalactic medium does indeed achieve a numerically exact and time invariant representation of the anomalous galactic star redshift without any appreciable Doppler contribution (Figure 6).

The above studies appear to provide sufficient experimental evidence acquired on Earth on the interior character of the large scale structure of the universe. In the next section, we shall show corresponding experimental evidence on the interior character of the structure of hadrons, nuclei and stars, thus suggesting the need to study interior problems for both the large and small scale structures of the universe. 


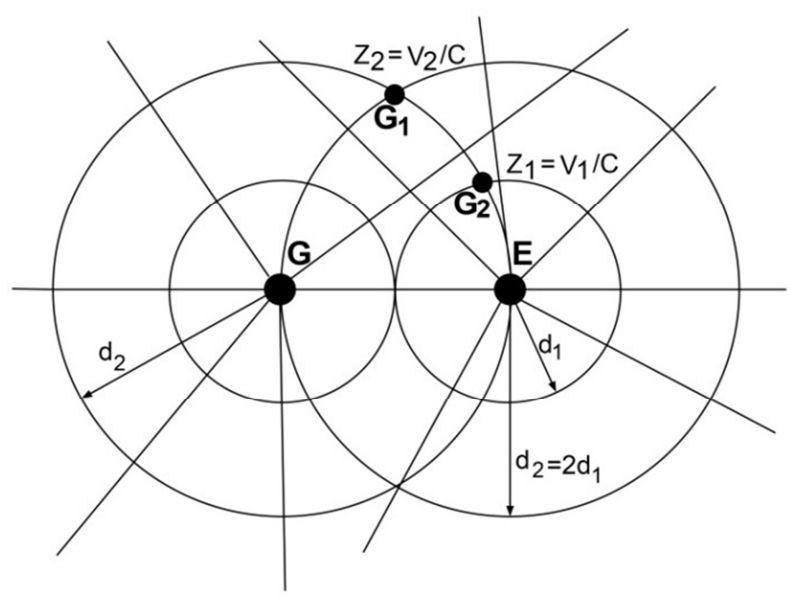

Figure 4. An illustration of the geometric inconsistency of all possible models on the expansion of the universe because galaxies $G_{1}$ and $G_{2}$ have a relative acceleration when seen from Earth $E$ due to the double distance, while there exists an infinite number of observers in the universe for which the same galaxies $G_{1}$ and $G_{2}$ have the same distance, as it is the case for galaxy $G$, in which case there is no relative acceleration, with ensuing clear inconsistency of the very conjecture of the expansion of the universe [43-47].

\section{Maximal Speeds for Interior Problems Within Physical Media}

The author has essentially devoted fifty years of research to the mathematical, theoretical and experimental studies of interior dynamical problems beginning with his $\mathrm{Ph}$. D. theses in the 1960s [9]. The general irreversibility over time of interior dynamical problems has requested the introduction since the mid 1960s of the Lie-admissible covering of Lie's theory which is at the foundation of the 20th century interpretation of special relativity [10-16].

The covering Lie-admissible formulations admit a particular case known as Santilli LieOisotopic formulations, that apply for interior dynamical problems when considered as isolated from the rest of the universe, thus being reversible over time [23-34].

The conceptual foundations of these studies can be summarized as follows: when elementary particles move in vacuum as empty space, their only possible acceleration is that via action-at-a-distance potential interactions (more technically known as variationally selfadjoint (SA) interactions [15a]). In this case, it is easy to see that the achievement in vacuum of the speed of light $c$ requires infinite energy and, therefore, the surpassing of the speed $c$ in vacuum by ordinary masses or electromagnetic waves is excluded.

However, Santilli [10] showed in 1981 that the situation is substantially different when elementary particles move in interior conditions because, in this case, accelerations are the result, not only of conventional SA interactions, but also of contact non-potential interactions (technically known as variationally nonselfadjoint (NSA) interactions, [15a]) for which the notion of "potential energy" has no physical value or meaning.

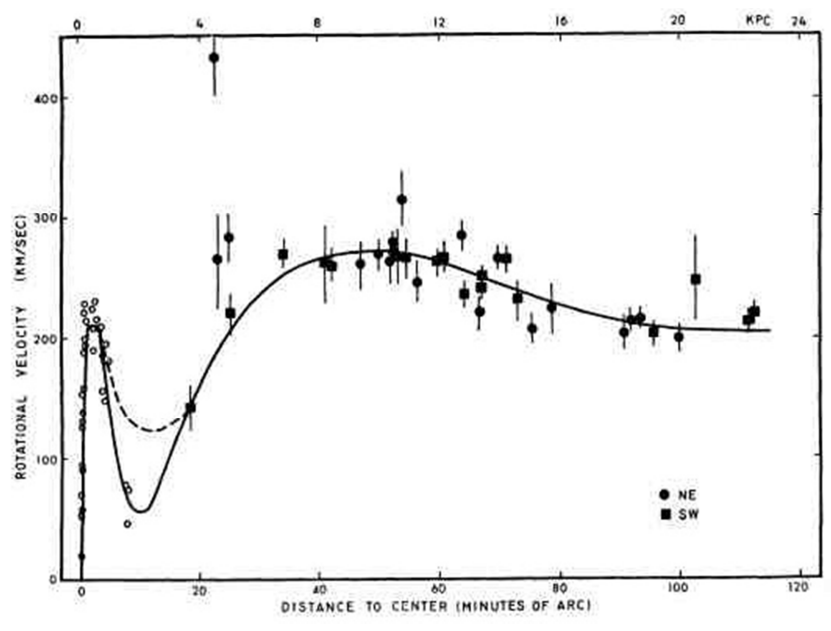

Figure 5. A plot of the anomalous redshift of galactic stars showing the decrease of the redshift for stars near the galactic center and its increase for peripheral stars. The conjecture of dark matter was ventured to represent theanomalous galactic stars, but no such a quantitative representation has appeared in the refereed literature to date, besides intrinsic in consistencies the conjecture of dark matter identified in Refs. [43-47].

It was then easy to see already in the 1980s that under NSA interactions the local speeds of ordinary masses within physical media are unrestricted, thus being arbitrarily bigger (or smaller) than $c$ depending on local conditions of density, temperature, frequencies and other physical data.

The analytic background of the studies on interior conditions is given by the "true Hamilton's equations," those with external terms not derivable from a Hamiltonian

$$
\frac{d r}{d t}=\frac{\partial H(r, p)}{\partial p}, \frac{d p}{d t}=-\frac{\partial H(r, p)}{\partial r}+F^{N S A}(t, r, v, \ldots),
$$

and their operator counterpart, which are specifically set for the representation of open irreversible processes we cannot possibly review here [16].

The mathematical backgrounds of the studies is given by the Lie-admissible covering of Lie's theory since the true Hamilton's equations emerged since the 1960s [9] as admitting a Lie-admissible algebra in the brackets of their time evolution when properly written (see the more recent memoir [13] for details).

To understand the complexity of the problem, let us recall that physical theories can be claimed to have physical value if and only if they verify the invariance over time, namely, they predict the same numerical values under the same conditions at different times. It is easy to see that the true Hamilton's equations and their operator counterpart violate this crucial condition because they are non-canonical and non-unitary by conception.

The achievement of invariance over time for non-canonical and non-unitary theories required the construction of a new mathematics, today known as Lie-admissible genomathematics we cannot possibly review here (see mathematics studies [11-13] and monographs [16]). 


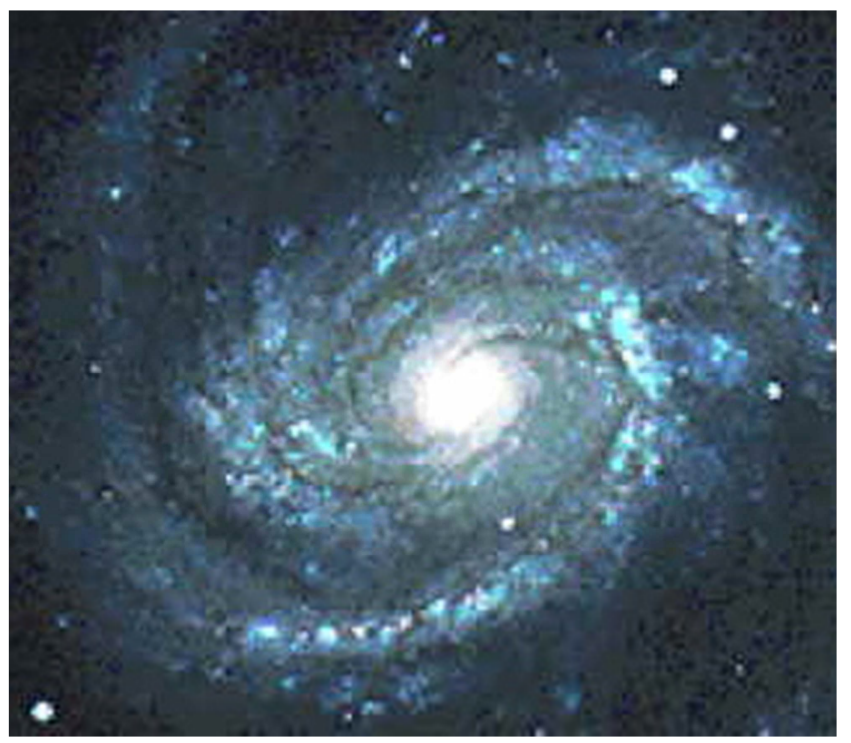

Figure 6. An illustration of the evidence visible via telescopes that all galaxies are filled up indeed of matter, but of a conventional, real, gaseous medium which is very hot near the galactic center and very cold for peripheral stars. The deviations from the conventional interpretation of special relativity measured by Santilli's isoblueshift for hot gases and isoredshift for cold gases [43-45] has permitted a numerically exact and invariant representation of the anomalous redshift of galactic stars depicted in Figure 5 [46,47]. This and other representations, such as that of Arp's pair of connected quasars and galaxies with largely different cosmological redshifts, establishes that the large scale structure of the universe is an interior dynamical problem in which all cosmological redshifts are reduced to the experimentally established loss (absorption) of energy to (from) cold (hot) intergalactic or innergalactic media without any appreciable contributions from the Doppler's shift [43-47].

A main application of these studies has been the first achievement at both nonrelativistic and relativistic levels of an exact representation of all characteristics of the neutron in its synthesis insider stars according to Rutherford's "compression of the hydrogen atom," namely, from a proton and an electron according to the known reaction (for brevity, see review [14])

$$
p^{+}+e^{-} \rightarrow n+v,
$$

The main technical difficulty was due to the fact that the rest energy of the neutron is $0.872 \mathrm{MeV}$ bigger than the sum of the rest energies of the proton and of the electron, under which condition we would need "positive binding energies" which are anathema for quantum mechanics, since they cause the physical inconsistency of the Schrödinger equation.

Santilli's main point is that, even though there exist indeed particles with "point-like charges" (such as the electron), there exist no "point-like wavepackets" in nature. Therefore, Rutherford's compression of the extended wavepacket of the electron within the hyperdense medium inside the proton generates NSA interactions under which a solution of synthesis (7) has been indeed found [14].

The main mechanism is that contact interactions are NOSA and, therefore, they are non-unitary. The non-unitary image of Schrödinger equation achieves consistency under "positive binding energies" thanks to a new renormalization of the rest energies of the constituents (called isorenormalization) [14,
$16,34])$.

The aspect important for this paper is that an apparent necessary condition for the representation of all characteristics of the neutron in synthesis (7) is that the constituents of the neutron travel at (tangential) superluminal speeds.

Intriguingly, systematic plots of experimental data in hadron physics conducted in monograph [16d] without the aprioristic assumption of the Lorentz symmetry have confirmed superluminal speeds within the interior of hadrons in numerous cases, such as: phenomenological fits via gauge theories; anomalous meanlives of unstable hadrons with speed; the Bose-Einstein correlation; and other cases directly relevant for the study of superluminal speeds.

We regret not being able to review these phenomenological fits and related literature to avoid a prohibitive length of this paper. Nevertheless, their knowledge is important for a technical understanding of this paper.

We should add that, as clarified by Wall [17], the superluminal speeds of hadronic constituents here considered are not referred to tachyons as conventionally defined (see, e,g, Ref. [42]) because, as we shall see in the next sections, interior physical media cause a deformation (called "mutation") of the light cone with superluminal speeds of real valued masses. Therefore, the existence of tachyons (called in the field of this paper isotachyons) is shifted for speed beyond the maximal causal speed within physical media which are generally bigger than $c$, as shown in the next section.

More specifically, we are not excluding possible tachyonic contributions in the structure of hadrons or in other physical conditions [42]. The only point we would like to clarify is that, under the validity of isotopic theories for the hadronic structure, the speed characterizing tachyons has to be shifted beyond $c$ (see, later on, Eq. (21) and related arguments).

Independently from Santilli's research, additional relevant studies on superluminal speeds are the experimental works initiated in 1992 by Enders and Nimtz [18] (see also the more recent paper [19] for additional references and paper [20] ) suggesting apparent superluminal propagation of electromagnetic waves within certain physical guides.

The reconciliation of superluminal speeds with special relativity limit (4) is generally attempted by assuming that we are dealing with a "tunnel effect." However, in our view, tunnel effects generally refer to passages through a barrier, thus for distances of $1 \mathrm{fm}$ covered by the uncertainty principle, and not for travel over lengths of several meters, as occurring for Refs. [18-20].

Hence, it is well possible that, in reality, Refs. [18-20] deal with an interior dynamical problem in which case superluminal speeds are due to NSA interactions of electromagnetic waves with the guides (including the so-called "stray fields" that are known to be NSA) under which interactions superluminal speeds are quite natural. Additional cases of superluminal speeds of ordinary masses can also be treated as interior dynamical problems, but we regret not being able to treat them here for brevity.

Yet an additional case relevant for this paper is the recent 
controversy at CERN on superluminal or sub-luminal neutrinos, since the original 2011 announcements [21] indicated the detection of superluminal neutrino speeds, while the subsequent paper [22] indicated subluminal speeds.

We would like to point out that the truly fundamental issue for Refs. [21, 22] is the identification of a causal and time invariant formulation of particle motion in interior conditions, since for both views [21, 22] neutrinos travel underground from CERN to the Gran Sasso Laboratory.

The orthodox position is that neutrinos are point-like and travel underground without collisions, for which view interior conditions do not exist, and special relativity applies exactly.

However, there exist no point-like wavepackets in nature; to be physical, neutrinos must have an extended wavepacket that does interact with the wavepackets of peripheral atomic electrons in the dense underground conditions; special relativity cannot be even marginally formulated undergrounds., e.g., because light does not propagate there; and, therefore, the interior conditions of experiments [21, 22] are unavoidable on serious scientific grounds.

In the absence of the appropriate basic theory, the underground speed of neutrinos remains unsettled because the slightest modification of any form factor, parameter or data elaboration can produce subluminal results in Ref. [21] and superluminal results in Ref. [22], as experts in the field can verify.

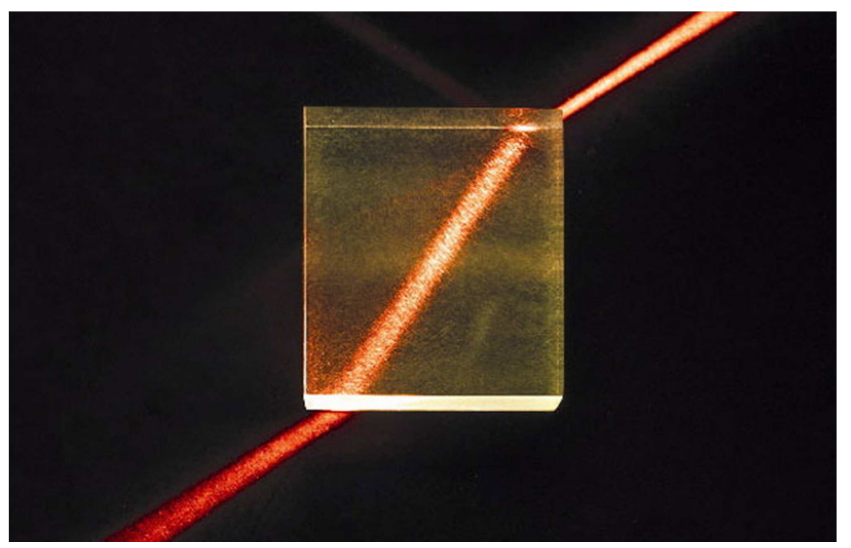

Figure 7. An illustration of the historical Lorentz problem at the foundation of this paper, the invariance of locally varying speeds of light within transparent physical media, here illustrated with the variation of speed from air to water and then back to air. The orthodox view is that light can be reduced to photons scattering among the water molecules, thus propagating in vacuum without any interior conditions. However, such an abstraction is known not to represent experimental data such as: the angle of refraction (because photons will scatter in all directions at the impact with water); the large reduction of speed for about 1/3 (because photons could at best account for a few percentages of speed reduction); the propagation of light in water as a straight beam (because photons would scatter light in all directions and the view of this picture would be impossible; and other experimental data. Hence, on serious scientific grounds, the representation of the propagation of light within transparent physical media requires a covering of special relativity specifically conceived for interior conditions.

Besides superluminal speeds, the study of interior dynamical problems inevitably requires the inclusion of subluminal speeds, namely, speeds of electromagnetic waves within transparent physical media smaller than $c$. This is typically the case for the propagation in water of infrared or radio waves for which the reduction to photon is not possible (Figure 1) [16a].

In this paper, we shall show that, when formulated with the appropriate mathematics, Special relativity axioms do indeed admit fully causal and time invariant arbitrary, that is whether superluminal or subluminal speeds depending on the physical characteristics of the medium at hand.

Since the Lie-admissible genomathematics is excessively advanced for the limited scope of this paper, we shall restrict our study to the particular case of interior events that are reversible over time, namely, events in which we ignore in first approximation the dispersion of light (Figure 1). This class of reversible interior events can be well treated via isomathematics, with particular reference to isorelativity, referred to the Lie-isotopic genomathematics of special relativity.

Nevertheless, the reader should keep in mind that the propagation of light within transparent physical media is an intrinsically irreversible event, as established by the simple evidence that media become warmer when traversed by light. Consequently, we should stress that the isotopic formulations presented in this paper only permit a first approximation of interior conditions.

That the proper causal and time invariant representation of irreversible interior events requires the covering genomathematics, and related genorelativity, referred to the Lie-admissible covering of isorelativity. To avoid a prohibitive length, this broader treatment will be merely indicated for intended presentations in subsequent papers.

We should finally recall historical contributions [67-71] dealing with superluminal speeds (although not within the context of interior dynamical problems),

\section{Solution of the Historical Lorentz Problem}

As it is well known to physics historians, Lorentz first attempted the achievement of the invariance of the speed of electromagnetic waves of his time, namely, the locally varying speed within physical media here referred to infrared, radio and other large wavelengths not admitting a consistent reduction to photons (see Section 4 for the general case)

$$
C=\frac{c}{n(t, r, v, e, \rho, \omega, \tau, \ldots)}
$$

where $n$ is the familiar index of refraction with a rather complex functional dependence on local variables, such as time $t$, coordinates $r$, speeds $v$, energy $e$, density $\rho$, frequency $\omega$, temperature $\tau$ and other variables.

Due to insurmountable technical difficulties, Lorentz was solely able to achieve invariance for the constant speed $c$ of electromagnetic waves in vacuum, resulting in the celebrated transformations (1) leaving invariant line element (2a).

Santilli has studied for decades the solution of the historical Lorentz problem, namely, the achievement of the universal 
invariance of all possible locally varying speeds of electromagnetic waves within physical media, Eq. (8), which case evidently admits the constant speed $c$ in vacuum as a particular case.

As a first step, when a member of MIT from 1974 to 1978 , Santilli realized that Lorentz's inability to achieve the invariance of speeds (8) was due to insufficiencies of the basic theory, Lie's theory, because such a theory is strictly linear, local-differential and potential-Hamiltonian, while the invariance of speeds (8) is a strictly non-linear, non-local/integral and non-potential, thus non-Hamiltonian problem.

The results of these initial studies were released in monographs [15] (that originally appeared as MIT preprints to be subsequently published under affiliation to Harvard University). A main aspect of these studies is their conception as isotopic (intended in the Greek meaning of being "axiom preserving") lifting of the various branches of Lie's theory into such a form to admit the treatment of non-linear, non-local and non-Hamiltonian systems.

The proposal was centered in the isotopic lifting of the unit of the Lorentz symmetry, $I=\operatorname{Diag} .(1,1,1,1)$, into a quantity $\hat{I}$ (such as a function, a matrix, an operator, etc.) with an arbitrary functional dependence on all needed local variables, under the sole condition of being positive-definite, thus invertible,

$$
\begin{gathered}
I=\operatorname{Diag} \cdot(1,1,1,1) \rightarrow \hat{I}=\hat{I}(t, r, v, e, \rho, \omega, \tau, \ldots)= \\
1 / \hat{T}(t, r, v, \rho, \omega, \tau, \ldots)>0,
\end{gathered}
$$

which lifting remains fixed for the interior problem considered.

For consistency, the lifting of the unit required the compatible lifting of the conventional associative product between arbitrary quantities $A$ and $B$ of the type

$$
A B \rightarrow A \widehat{\times} B=A \widehat{T} B,
$$

under which $\hat{I}$ is indeed to the right and left unit of the theory,

$$
\hat{I} \widehat{\times} A=A \widehat{\times} \hat{I} \equiv A,
$$

for all elements $A$ of the set considered.

Following basic assumptions (9)-(11), Santilli constructed a step by step isotopic generalization of the various branches of Lie's theory, including [15b]:

1) The isotopic lifting $\hat{\xi}(L)$ of the universal enveloping associative algebra $\xi(L)$ of a $n$-dimensional Lie algebra $L$ with (Hermitean) generators $X_{i}, i=1,2, \ldots, n$, and infinite-dimensional isotopic basis (today known as the Poincaré-Birkhoff-Witt-Santilli isotheorem [35-42]):

$$
\hat{I}, X_{k}, \hat{X}_{i} \widehat{\times} \hat{X}_{j}, i \leq j, \hat{X}_{i} \widehat{\times} \hat{X}_{j} \widehat{\times} \hat{X}_{k}, i \leq j \leq k, \ldots \text {; }
$$

2) The isotopic liftings of Lie algebras with closure rules (today called Lie-Santilli isoalgebras [loc. cit.]

$$
\left[X_{i}, X_{j}\right]=X_{i} \widehat{\times} X_{j}-X_{j} \widehat{\times} X_{i}=C_{i j}^{k} X_{k}{ }^{\prime}
$$

3) The corresponding isotopic lifting of Lie's transformation groups (today called Lie-Santilli isogroups [loc. cit.]), e.g., here expressed for the time evolution

$$
A(t)=U(t) A(0) U(t)^{\dagger}=\left[e^{H \hat{T} t i}\right] A(0)\left[e^{-i t \hat{T} H}\right] ;
$$

and the isotopies of the representation theory.

The above isotopies clearly show the non-linear, non-local (integral) and non-Hamiltonian character of the isotopic theory due to the appearance of a positive-definite but otherwise arbitrary quantity $\hat{T}$ in the exponent of the group structure.

The representation of interior systems is then achieved via the representation of all SA interactions by means of the conventional Hamiltonian $H(r, p)$, and the representation of all NSA interactions by means of the generalized unit $\hat{I}$ (see Refs. $[16,34]$ for concrete examples in classical and operator mechanics).

Following the construction of the isotopies of Lie's theory, Santilli introduced in letter [23] of 1983 the following isotopies of Minkowski space (2) (today known as the Minkowski-Santilli isospace [loc. cit.]) with the most general possible nonsingular and symmetric line element (thus including all possible Minkowskian, Riemannian, Fynslerian and other line elements in (3+1)-dimensions)

$$
\begin{gathered}
\widehat{x}^{\widehat{2}}=x^{\mu}\left(\widehat{T}_{\mu}^{\rho} \eta_{\rho v} x^{v}=x^{\mu} \hat{\eta}_{\mu v} x^{v}=\frac{x_{1}^{2}}{n_{1}^{2}}+\frac{x_{2}^{2}}{n_{2}^{2}}+\frac{x_{3}^{2}}{n_{3}^{2}}-t^{2} \frac{c^{2}}{n_{4}^{2}}\right. \\
n_{\mu}=n_{\mu}(t, r, v, e, \rho, \omega, \tau, \ldots)>0, \mu=1,2,3,4 \\
\hat{T}=\operatorname{Diag} \cdot\left(1 / n_{1}^{2}, 1 / n_{2}^{2}, 1 / n_{3}^{2}, 1 / n_{4}^{2}\right)>0 \\
\hat{I}=1 / \hat{T}=\operatorname{Diag} \cdot\left(n_{1}^{2}, n_{2}^{2}, n_{3}^{2}, n_{4}^{2}\right)>0
\end{gathered}
$$

where: the $n$ 's are called the characteristic quantities of the medium considered; $n_{4}$ is the conventional index of refraction providing a geometrization of the density of the medium normalized to the value $n_{4}=1$ for the vacuum; $n_{1}, n_{2}, n_{3}$ provide a geometrization of the shape of the medium considered normalized to the values $n_{1}=n_{2}, n_{3}=1$ for the sphere; the general inhomogeneity of the medium is represented by the dependence of the characteristic quantity on the local variables (e.g., the elevation for the case of our atmosphere); and the general anisotropy of the medium (e.g., the anisotropy of our atmosphere caused by Earth's rotation) is represented by different values of the type $n_{4} \neq n_{s} .{ }^{1}$

Note that, for exterior dynamical problems, homogeneity and isotropy equally occur in all directions. By contrast, within a physical medium inhomogeneity and anisotropy requires the selected of a given direction in space $n_{s}$ due to variations for different directions.

Following the construction of the isotopies of Lie's theory and of Minkowski space-time, Santilli solved the historical Lorentz problem in page 551 of letter [23] via the lifting of the

\footnotetext{
${ }^{1}$ Scientific caution is suggested before dubbing the $n$-characteristic quantities as "free parameters" because that would imply that, e.g., the index of refraction $n_{4}$ is a free parameter when in reality it is measured for a given medium, or that the $g_{u \mu}$ elements of the Schwartzchild metric (see Eqs. (17) below) are free parameters, etc.
} 
Lorentz symmetry characterized by the isotopic element (15c). This resulted in the generalized transformations (Eqs. (15) of Ref. [23]), today known as the Lorentz-Santilli (LS) isotransforms [35-42] which we write in the currently used symmetrized form

$$
\begin{gathered}
x^{` 1}=x^{1}, x^{` 2}=x^{2}, \\
x^{` 3}=\hat{\gamma}\left(x^{3}-\hat{\beta} \frac{n_{3}}{n_{4}} x^{4}\right), x^{` 4}=\hat{\gamma}\left(x^{4}-\hat{\beta} \frac{n_{4}}{n_{3}} x^{3}\right), \\
\hat{\beta}=\frac{v_{3} / n_{3}}{c_{o} / n_{4}}, \hat{\gamma}=\frac{1}{\sqrt{1-\widehat{\beta}^{2}}}
\end{gathered}
$$

leaving invariant the isoline element (15a), thus providing indeed the invariance of the varying speeds of light (8) (see Ref. [34b] for the general treatment).

Jointly with the above classical formulation, Santilli constructed the corresponding operator image [24] of the above isotransformations, and then constructed the isotopies of every main aspect of the LP symmetry, including the isotopies of: the rotational symmetry [25]; the $\mathrm{SU}(2)$-spin symmetry [26]; the Poincaré symmetry [27]; the spinorial covering of the Poincaré symmetry [28]; the SU(2)-isospin symmetry and local realism [29]; and the isotopies of the Minkowskian geometry [30].

The resulting isosymmetry, today known as the Lorentz-Poincaré-Santilli (LPS) isosymmetry, was proved in Refs. [31,32] to be "directly universal" for all infinitely possible non-singular and symmetric space-times in $(3+$ 1)-dimensions, thus providing the universal symmetry of all possible Riemannian, Fynslerian, and other possible line elements, with a trivial extension to arbitrary space-time dimensions, such as those for the De Sitter symmetry.

Systematic studies can be found in monographs [33, 34], while independent studies can be found in monographs [35-42] and references quoted therein. A few comments are now in order to prevent possible misrepresentations that generally remain undetected by non-experts in the field.

To illustrate the universality of isoinvariant (15) and related isosymmetry (16) for all possible, symmetric and non-singular space-times in $(3+1)$-dimensions, let us note that they include as particular case all possible Riemannian line elements, such as the Schwartzchild's line element [27]

$$
\begin{array}{r}
d s^{2}=r^{2}\left(d \theta^{2}+\sin ^{2} d \theta^{2}+d \phi^{2}\right)+\left(1-\frac{2 \times M}{r}\right)^{-1} \times d r^{2}- \\
\left(1-\frac{2 \times M}{r}\right) \times d t^{2} \\
\hat{T}_{\text {sch }}=\text { Diag. }\left[1,1,\left(1-\frac{2 \times M}{r}\right)_{r}^{-1},\left(1-\frac{2 \times M}{r}\right)_{t}\right], \quad(17 \mathrm{~b}) \\
\hat{I}_{s c h}=\text { Diag. }\left[1,1,\left(1-\frac{2 \times M}{r}\right)_{r},\left(1-\frac{2 \times M}{r}\right)_{t}^{-1}\right], \quad \text { (17c) }
\end{array}
$$

where one can see that the gravitational singularity is that of the isotopy, namely, the infinite value of the isotopic element and the null value of the isounit. In fact, Ref. [27] was primarily intended to indicate the achievement of the universal symmetry for all possible (non-singular) Riemannian line elements.
A rather popular belief during the 20th century physics was that interior dynamical systems are not essential because they can be reduced to elementary particles moving in vacuum, thus recovering at the elementary level exterior conditions without non-linear, non-local and non-Hamiltonian interactions. This belief was disproved by the following:

NO REDUCTION THEOREM [13, 34]: A macroscopic, non-conservative system cannot be consistently reduced to a finite number of elementary particles all in conservative conditions and, vice versa, a finite number of elementary particles all in conservative conditions cannot consistently yield a macroscopic non-conservative system under the correspondence or other principles.

Stated in different terms, the reduction of interior to exterior systems implies the belief that entropy and thermodynamical laws are "illusory" because, when interior systems are reduced to elementary particle constituents, entropy and thermodynamical laws "disappear." The above No Reduction Theorem establishes that non-conservative (thus, NSA) interactions, rather than "disappearing," originate at the most elementary level of nature.

As a concrete example, the above No Reduction Theorem is verified by a spaceship during reentry in atmosphere because its non-linear, non-local and non-Hamiltonian interactions originate at the most elementary level, that of the deep mutual penetration of the wavepackets of peripheral atomic electrons of the spaceship with the wavepackets of the electrons of atmospheric atoms.

The next aspect needed for a serious understanding of the content of this paper is the necessity to verify the time invariance indicated in the preceding section, namely, the prediction of the same numbers under the same conditions at different times.

Santilli selected a generalization of the unit for the representation of non-linear, non-local and non-Hamiltonian interactions for the intent of achieving the much needed time invariance, since the unit is the basic invariant of any theory.

However, it is easy to see that the representation of non-Hamiltonian interactions via the isounit is not sufficient per se to achieve the needed time invariance because isotopic theories are non-canonical at the classical level and non-unitary at the operator level by conception and construction $[16,33,34]$. It is then easy to see that the isounit is not preserved by the time evolution of the theory, e.g., Eq. (14)

$$
\hat{I} \rightarrow \hat{I}^{\prime}=U \hat{I} U^{\dagger} \neq \hat{I}, U U^{\dagger} \neq I
$$

But the isounit represent interior conditions. Therefore, its lack of conservation in time implies the transition over time from one interior system to another (e.g., from the synthesis of the neutron (7), for instance, to a nuclear fusion).

This occurrence is known under the name of Theorem of Catastrophic Mathematical and Physical Inconsistencies of Non-Canonical and Non-Unitary Theories when formulated with the mathematics of canonical and unitary theories, respectively. Regrettably, we cannot review this theorem for brevity and must refer the reader to works [13, 33, 34].

The resolution of the inconsistencies caused by the lack of 
time invariance required decades of additional research by Santilli and a number of colleagues. The solution was finally achieved following the construction of a new mathematics, today known as Santilli isomathematics, characterized by the isotopic lifting of the totality of the quantities and their operation of the mathematics used for exterior problems.

When classical non-canonical and operator non-unitary theories are elaborated with the appropriate classical and operator isomathematics, respectively, the invariance over time of numerical predictions is regained, thus offering the mathematical and physical consistency needed for applications.

\section{Compatibility of Arbitrary Interior Speeds with Special Relativity}

The locally varying speeds of electromagnetic waves propagating within physical media left invariant by the LPS isosymmetry (16) are completely unrestricted and can, therefore, be smaller, equal or bigger than the speed of light in vacuum,

$$
C=\frac{c}{n_{4}}<=>c
$$

This is due to the unrestricted functional dependence of the isotopic element (15c), except for the condition of being non-singular.

It is then easy to see that the maximal causal speed in Minkowski-Santilli isospaces is arbitrarily bigger, equal or smaller than $c$. In fact, the mutated light cone, called light isocone, in the $(s, 4)$-dimensions is given by

$$
\widehat{x}^{\widehat{2}}=\frac{x_{s}^{2}}{n_{s}^{2}}-t^{2} \frac{c^{2}}{n_{4}^{2}}=0
$$

and evidently characterizes the maximal causal speed within physical media

$$
V_{\text {max }, s}^{\text {media }}=c \frac{n_{s}}{n_{4}}<=>c .
$$

where, as indicated in Section 1, the selection of a space direction $s$ is necessary since physical media are generally inhomogeneous and anisotropic.

Note the need to use a covering of the speed of light for maximal causal speed under the LPS isosymmetry because interior dynamical problems are generally opaque to light. Note also that the causal character of speeds (21) is guaranteed by the LPS isosymmetry in exactly the same way as the LP symmetry guarantees the causal character of $c$.

It has been popularly believed throughout the 20th century that any deviation from the speed of electromagnetic waves in vacuum implies a "violation of Einstein's special relativity." In this section, we show that this belief is not technically correct, because Einstein's special relativity axioms do admit arbitrary causal speeds, provided that they are realized via the appropriate mathematics.

To begin, Lorentz transformations (1) provide the invariance of the "constant" speed $c$ without any identification of its numerical value, which value is set by measurements. Therefore, Lorentz transformations equally apply for an arbitrary constant speed $C=c / n_{4}$ within physical media, with known value in water

$$
C_{\text {water }}=\frac{c}{n_{4}}=\frac{2}{3} c, n_{4}=\frac{3}{2} .
$$

in which case no violation of Einstein special relativity axioms can be claimed.

Additionally, the replacement in the conventional transforms (1) of the speed of light $c$ with maximal causal speed (21) yields, identically, the LPS isotransformations (16), as one can readily verify.

$$
\begin{aligned}
& x^{{ }^{1}}=x^{1}, x^{` 2}=x^{2}, \\
& x^{` 3}=\gamma\left(x^{3}-\beta x^{4}\right), \\
& x^{{ }^{4}}=\gamma\left(x^{4}-\beta x^{3}\right), \\
& \beta=\frac{v}{V_{\text {max }}^{\text {media }}}, \gamma=\frac{1}{\sqrt{1-\beta^{2}}} .
\end{aligned}
$$

Also, the Lorentz-Santilli isosymmetry (16) is locally isomorphic to the conventional Lorentz symmetry by conception and construction to the point of preserving the original structure constants [23, 33, 34]. Therefore, no claim that isotransforms (16) violate Einstein's special relativity axioms can be consistently voiced due to the very conception and technical realization of the isotopies of special relativity.

More technically, when represented on Minkowski-Santilli isospace over the isofield of real numbers [11], light isocone (20) becomes the perfect cone with the same maximal causal speed $c$ as that valid in empty space $[33,34] .^{2}$

This is due to the fact that the cone axes are indeed mutated under isotopies from their original unit value to new values

$$
\left(1_{s}, 1_{4}\right) \rightarrow\left(1 / n_{s}^{2}, 1 / n_{4}^{2}\right),
$$

but, jointly, the related units are mutated by the inverse amounts,

$$
\left(1_{s}, 1_{4}\right) \rightarrow\left(n_{s}^{2}, n_{4}^{2}\right),
$$

thus preserving the original Minkowskian light cone identically.

We finally illustrate the compatibility of arbitrary speeds with special relativity axioms via the following transformation of the Minkowskian coordinates

$$
x^{\mu} \rightarrow \frac{x^{\prime \mu}}{n_{\mu}}
$$

under which the Minkowski line element (2a) is transformed into the isotopic image (15a), and Lorentz transformations (1) are turned into the LS transform by keeping in mind transformation of the speed, e.g., along the third space

\footnotetext{
${ }^{2}$ The mathematically correct formulation of the Minkowski-Santilli isospace is given by the isospace $\widehat{M}(\hat{x}, \hat{\eta}, \hat{I})$ defined over the field of isoreal numbers with unit $\hat{I}$ given by Eq. (15c), with $\hat{x}=x \hat{I}$ as a condition to be an isonumber and $\hat{\eta}=\hat{T} \eta$ (see Ref. [30] for details).
} 
direction

$$
v=\frac{\delta x^{3}}{\delta t} \rightarrow v \frac{n_{4}}{n_{3}} .
$$

By recalling that the metric of isotopic theories $\hat{\eta}=\hat{T} \eta$ includes as particular case all possible Riemannian metrics $g$, in this section we have attempted to indicate that Einstein's special relativity axioms have a representational capability dramatically broader than that believed in the 20th century because, in addition to admitting arbitrary maximal causal speeds, they also admit interior and exterior gravitational models.

In fact, in Ref. [30] Santilli has shown the treatment of exterior gravitation via special relativity axioms on the metric $\hat{\eta}(x)=\hat{T}(x) \eta=g(x)$, while maintaining the machinery of the Riemannian geometry (covariant derivative, Christoffel's symbols, etc.) and Einstein-Hilbert field equations under the universal LPS symmetry as a condition to achieve the above indicated invariance over time of numerical predictions. In this exterior case, the maximal causal speed is evidently that in vacuum $c$.

In the same Ref. [3], Santilli has shown that special relativity axioms equally admit interior gravitational models, this time, with an unrestricted functional dependence of the metric $\quad \hat{\eta}(x, \rho,-\tau, \omega, \ldots)=\widehat{T}(x, \rho, \tau, \omega, \ldots) \eta=$ $g(x, \rho, \tau, \omega, \ldots)$ equally under the universal LPS symmetry, in which case the maximal local causal speed is arbitrarily bigger or smaller than $c$ depending on local conditions.

Note that, under the full use of isomathematics, all the preceding enlargements of the conditions of applicability of Einstein's special relativity axioms can be formulated via conventional symbols as used in Eqs. (1)-(4), and merely subject them to different interpretations.

In fact, the variables $x^{\mu}$ can be interpreted as representing physical space-time coordinates with respect to the Lorentz unit $I=$ Diag. $(1,1,1,1)$, in which case we have the 20th century formulation of special relativity for exterior problems invacuum.

Alternatively, we can consider the coordinates $x^{\mu}$ as being purely mathematical and assume the realization $x^{\mu}=x^{\prime \mu} / n_{\mu}$. In this case, when $x^{\prime \mu}$ are assumed as the physical coordinates and are referred to the isounit (15d), we realize special relativity axioms in such a way to have interior dynamical conditions with maximal causal speeds 921), exterior gravitation with the characteristic quantities representing conventional Riemannian metrics, interior gravitation, and other possibilities.

It should be stressed that the above studies apply to interior dynamical problems that are reversible over time. Their extension to irreversible problems can be first studied via isounit with an explicit but not invariant time dependence of the type

$$
\hat{I}(t, \ldots)-\hat{I}^{\dagger}(t \ldots) \neq \hat{I}(-t, \ldots)=\hat{I}^{\dagger}(-t, \ldots),
$$

The reader should however be aware that under the above realization of the isounit the Lie-isotopic formulation is turned into a bimodular formulation with a Lie-admissible structure
[9-16].

We should finally indicate that the entire body of mathematical, theoretical and experimental formulations studied in this paper are nowadays known under the name of isorelativity for matter [50-54] and the isodual isorelativity for antimatter[55].

\section{Expected Implications}

\subsection{Cosmology}

As reviewed in Section 2, the conjecture of the expansion of the universe and its endless chain of subsequent conjectures were aimed at the unspoken intent (or de facto primary implication) of maintaining the validity of special relativity for the large scale structure of the universe because the alternative offered by Zwicky [5], that of galactic light losing energy to the intergalactic medium, would violate the special relativity.

Despite one century of efforts, the conjecture of the expansion of the universe is nowadays discredited in serious scientific circles due to excessive insufficiencies or sheer inconsistencies published in refereed journals, but none of them disproved also in refereed journals.

In fact, all current dominating cosmological models imply a return to the Middle Ages with Earth at the center of the universe due to the radial character in all directions from Earth of the conjectured expansion of the universe and its conjectured acceleration.

Isomathematics [38], the Lorentz-Santilli isosymmetry [23-30], its related isorelativity [51-54], and the vast experimental verifications on Earth of Zwicky hypothesis [43-47] are expected to restore the validity of special relativity for the large scale structure of the universe without inconsistent assumptions on the expansion of the universe.

This important occurrence is due to the fact that the Lorentz-Santilli isosymmetry uniquely and unambiguously characterize the Doppler-Santilli isoshift law [51, 43]

$$
z \approx \pm \frac{v}{c} \frac{n_{4}}{n_{v}}
$$

where the characteristic quantities $n_{4}, n_{v}$ depend on all local variables, including the speed $v$, the distance $d$ traveled by light, etc., thus allowing the expansion

$$
z \approx \pm \frac{v}{c} \frac{n_{4}}{n_{v}} \approx \pm \frac{v}{c}\left(1 \pm \frac{H c}{v} d\right)= \pm \frac{v}{c} \pm H d,
$$

where the first term is the conventional Doppler term, the second term is the Santilli isoredshift, and $H$ is a constant (in first approximation) that can be assumed to be Hubble's constant for cosmological applications.

But experiments [43-47] have established that the conventional Doppler contribution is ignorable with respect to Santilli isoredshift for the propagation of light within physical media either of large density and short distances as it is the case for our atmosphere, or of low density but extremely large distances, as it is the case for intergalactic media.

Consequently, the original, experimentally verified 
Hubble's law [4]

$$
z=H d
$$

is uniquely, unambiguously and invariantly represented by the Lorentz-Santilli isosymmetry. But the Lorentz-Santilli isosymmetry is locally isomorphic to the conventional symmetry, and the axioms of isorelativity are identical to those of special relativity [51-54], thus implying the restoration of special relativity for the large scale structure of the universe, although in its broader isotopic realization, without any expansion of the universe.

However, the cosmological implications of the above possibilities are far reaching because all current numerical values in cosmology, beginning with currently assumed distances of stars and galaxies, should be re-inspected for possible revisions whenever admitting the absorption of light by innergalactic or intergalactic physical media.

In fact, current distances are estimated based on the luminosity of supernovae or large stars under the current assumption, necessary to maintain special relativity in cosmology, that light propagates in vacuum.

It is evident that the admission of innergalactic and intergalactic media as well as the admission of the experimental evidence [43-47] on the loss of energy by light to said media, are expected to imply a revision of the value of the intensity at the origin, with consequential revision of current estimates on cosmological distances.

The same implications are expected for other numerical values in cosmology since they are all derived via theoretical conjectures, since the same experimentally known values are those of the cosmological redshift.

\subsection{Particle Physics}

The unspoken intent of maintaining special relativity in cosmology was also applied to the structure of strongly interacting particles (hadrons) via the same approach, that based on conjecture that could not be directly verified in experiments, so that the conjectures would achieve collegial acceptance via coordinated supports.

In fact, the structure of hadrons was reduced to the hypothetical quarks that, by conception have to be permanently confined inside hadrons, thus reducing the structure of hadrons to imaginary spheres containing point-like hypothetical quarks moving in vacuum, as necessary for the validity of special relativity.

In particular, for the first time in history, the same model was used for both the classification of hadrons into family and the structure of individual hadrons belonging to a given multiplet. By contrast, atoms required two models, the Mendeleev classification of atoms into families, and the structure of individual atoms belonging to a given family.

Also, the classification of atoms was achieved via classical methods, while the structure of individual atoms required the construction of a generalization of classical theories, quantum mechanics.

The insufficiencies of such an ad hoc conduction of physical research are numerous (see, e.g., paper [56] of 1981). Unfortunately for scientific knowledge, said insufficiencies are not disproved in refereed journals exactly as it has been the case for the insufficiencies of cosmological conjectures.

The most striking insufficiency with large societal implication is that belief, implicit in quark conjectures, that the permanently stable proton and electron "disappear" (sic) at the time of the synthesis of the neutron (7) top be replaced by the hypothetical quarks and, then, at the time of spontaneous decays of the neutron, the hypothetical quarks "disappear" and the permanently stable proton and electron "reappear."

Additionally, quarks cannot be defined in our spacetime due to incompatibilities with the Poincaré symmetry, as well known to quark experts. Therefore, any claim that quarks are "physical particles" has no serious physical ground. Additionally, the theoretical impossibility for quarks to be seriously confined inside hadrons (that is, to have an identical null probability of tunnel effects) is readily established by Heisenberg's uncertainty principle. In the final analysis, quarks are mere mathematical representations of a purely mathematical symmetry solely defined in a purely mathematical, unitary, complex-valued space.

In view of all the above insufficiencies (and numerous others), the view suggested by the author when at Harvard University in the 1980s [56] is that the $S U(3)$-color classification of hadrons (now extended to the "Standard Model") is excellent; quarks are necessary for the mathematical elaboration of said classification; and the structure of individual hadrons requires a generalization of quantum mechanics into hadronic mechanics.

The latter view was suggested in the early 1980s because there exist no point-like wavepackets in nature. Consequently, hadrons are the densest medium identified by mankind to date caused by the total mutual penetration of the wavepackets of the constituents, with inevitable non-linear, non-local and non-Hamiltonian internal effects. Under these conditions, the intents at preserving the 20th century formulation of special relativity and quantum mechanics are non-scientific, besides being disproved by experimental evidence on the behavior of the main-life of unstable Kaons from $1 q 0$ to $100 \mathrm{GeV}$ showing clear deviations from special relativity [57].

Isomathematics [38], the Lorentz-Santilli isosymmetry [23-30], and the additional experimental evidence on deviations from special relativity within physical media [43-47], offer realistic possibilities of restoring the validity within hadrons of the abstract axioms of special relativity and quantum mechanics, although in their generalized isotopic realizations, without the assumption of hypothetical constituents that have not been produced free even at the extremely high energies available at CERN.

According to this view, the constituents of all unstable particles are generally the massive particles produced in the spontaneous decays, although in a generalized form characterized by the Poincaré-Santilli isosymmetry under the law of relativistic hadronic mechanics due to their total mutual immersion (see review [42] and original papers quoted therein). 
As it was the case for cosmology, the implications of the above new vistas in particle physics are rather deep because they require the re-inspection of the entire 20th century particle physics for possible revision.

As an example, the Pauli-Fermi conjecture of the neutrino, voiced to conserved the angular momentum in synthesis (7), is fundamentally dependent on the abstraction of the proton as a point-particle because necessary for the very applicability of quantum mechanics, as it is well known.

The representation of the proton as an extended charge distribution has eliminated the need for the neutrino to conserve the angular momentum because, during the "Rutherford's compression" of the hydrogen atom inside a star, the electron is constrained to orbit within the hyperdense medium inside the proton in such a fashion to have a null total angular momentum, in which case the spin of the neutron in synthesis (7) is the spin of the proton [14].

In any case, the Pauli-Fermi conjecture of the neutrino did not salvage quantum mechanics because the rest energy of the neutron is $0.782 \mathrm{MeV}$ bigger than the sum of the rest energies of the proton and the electrons, in which case the Schrödingerequation is completely ineffective (for a bound states). The covering hadronic mechanics was proposed precisely for the representation of synthesis (7) and it proved to be correct at both the non-relativistic and relativistic level [14].

Despite the latter advances, our knowledge of the synthesis of the neutron remains Lilliputian at best and so much remains to be discovered by young minds of any age. For instance, we remain with the mystery of the origin of the missing $0.782 \mathrm{MeV}$ neededto create the neutron which energy cannot be due to relative kinetic energy (because in this case the cross section of the scattering of protons and electrons is virtually null).

The author has submitted the hypothesis that the missing energy originates from space conceived as a universal substratum for all electromagnetic waves and all particles with a verity high energy density (due to the high value of the speed of light). In this case, the energy is expected to be transmitted from space to the neutron via a longitudinal impulse the author has called the etherino, by turning the neutron synthesis into a Lie-admissible interior dynamical problem [58].

The aspect intriguing for this paper is that, being a longitudinal impulse in a universal medium with physical characteristics similar to that of "rigidity" (due to the transversal character of electromagnetic waves), the speed of transmission of energy from space to the neutron is expected to be a large multiple of the speed of light. This illustrates again the possible existence of arbitrary speeds for interior dynamical problems in a way fully compatible with the abstract axioms of special relativity and relativistic quantum mechanics, although realized via the covering Lie-isotopic and then Lie-admissible mathematics.

As a final comment, it appears that the etherino hypothesis can represent experimental data on the so-called "neutrino experiments" in a more credible way than that provides by contemporary neutrinos that are believed to have mass. In fact, the idea that massive particles can travel long distances within dense matter without collision is not plausible since a large number of neutrinos have to travel through a large number of nuclei without collision. By contrast, the same long travel through matter is more plausible for the etherino because the travel occurs within the underlying universe substratum, rather than through matter.

\subsection{Nuclear Physics}

The scientific scene depicted above for cosmology and particle physics occurred again in nuclear physics due to the restriction of nuclear physics research over one century to the unspoken (intent to be compatible with the conventional interpretation of special relativity and relativistic quantum mechanics despite truly vast insufficiencies.

In serious scientific circles, a theory can be assumed as being exactly valid for given, well defined conditions, if and only if said theory represents all experimental data from un-adulterated first principles. This has been indeed the case for the validity of quantum mechanics for the structure of the hydrogen atom.

Whenever a theory does not represent at least the main experimental data, said theory can be at best claimed to be approximately valid. In this conditions considered. Scientific ethics then requires the search for covering theories, as it was historically done for the atomic structure.

The conventional interpretation of special relativity and relativistic quantum mechanics can at best be considered as being approximately valid in nuclear physics because, following attempts over one century, they failed to achieve a consistent representation of the experimental data of the simplest nucleus, the deuteron, with embarrassing deviations for heavy nuclei such as the zirconium.

It is today known that the insufficiencies of standard theories in nuclear physics are due to the point-like abstraction of particles which is very effective for structures at large mutual distances of particles, such as the atomic structure, while being manifestly insufficient for the nuclear structure where the constituents are extended charge distributions in conditions of partial mutual penetration.

Isomathematics [38], the Lorentz-Santilli isosymmetry [23-30], and the experimental evidence on deviations from special relativity within physical media [43-47], offer realistic possibilities for new vistas in nuclear physics while preserving the abstract axioms of special relativity and quantum mechanics.

The central notion deals with the representation of nucleons as extended, therefore deformable charge distribution according to a conception dating back to Enrico Fermi, who indicated that the representation of nuclear magnetic moments may require the deformation of nucleons with consequential alteration of their magnetic moments. In view of their compatibility with the deformation theory [24-26], Santilli's Lie-isotopic formulations have provided a technical realization of Fermi's view, by achieving indeed the first known exact and invariant representations of nuclear magnetic moments $[59,60]$ and spins [61]. 
Independently from the above, a structural insufficiency of standard theories is their lack of a "time arrow," with the consequential sole capability of representing isolated stable bound states that, as such, are reversible over time. Consequently, quantum mechanics is structurally insufficient for the representation of nuclear energies, because they are all based on time irreversible processes. Santilli Lie-Isotopic and, more appropriately, Lie-Admissible reformulations of special relativity and quantum mechanics offer a realistic possibility for quantitative studies of new nuclear energies that are already under way at U. S, corporations [52].

A novel aspect important for this presentation is that the above new vista in nuclear physics is based, not only in the representation of nucleons as extended and deformable under partial mutual penetration, but also in the consequential emergence of a new component in the nuclear force of contact, non-Hamiltonian type invariantly represented by Santilli isounit. In turn, the sole emergence of this new component in the nuclear forces implies possible advances in nuclear physics simply beyond our appraisal capabilities at this writing.

\subsection{Chemistry}

The author has always accepted the historical advances permitted by quantum chemistry at molecular distances, but could not accept quantum chemistry for the valence bond because valence electron pairs in singlet couplings should repel, rather than attract each other, due to the extremely large values of their Coulomb repulsion which is of the order of $10^{28} \mathrm{~N}$ at bond distances of $1 \mathrm{fm}$.

It is today known that this insufficiency is due to the impossibility of a consistent reduction of the universe to exterior conditions, intended to be everywhere composed of point-particles moving in vacuum (empty space). Chemistry establishes alone the impossibility in view of the non-Hamiltonian interaction originating from the deep mutual penetration of the wavepackets of valence electrons establishing the valence bond is an interior dynamical problem.

In fact, said contact non-Hamiltonioan interactions have been able to overcome the repulsive Coulomb force and produce an attraction between the identical valence electrons in singlet coupling achieving an exact representation of the binding energies of the hydrogen and water molecules, as well as other chemical data [63].

Rather than being a mere academic curiosity, the achievement of an attractive force between valence electron pairs has permitted the identification of new bonds, called Santilli magnecular bonds, that, being weaker than the valence by conception and technical realization, allow full combustion, i.e., the absence of combustible contaminants in the exhaust such as CO, HT, etc., with evident environmental advances [64].

\subsection{Biology}

The insistence in the use of special relativity and quantum mechanics in biology implies that biological structures are perfectly rigid, evidently due to the known incompatibility of said theories with the deformation theory, and eternal, evidently due to the known lack of a time arrow in the basic axioms.

By contrast, the Lie-isotopic, Lie-admissible and hyper-structural coverings of special relativity and quantum mechanics [50] are compatible with the deformation theory by conception and technical realization [25-27], and contain a time arrow in their ultimate foundations, the basic units [65].

It is hoped that these new vistas may permit more realistic representations of biological structures with expected advances perhaps not entirely conceivable at this writing.

\subsection{Gravitation}

Recent studies [54] have reviewed the historical objection against Einstein's gravitation, with particular reference to:

1. The lack of experimental evidence on the actual curvature of space since the bending of star light passing near the Sun is entirely due to the refraction of light in the Sun chromosphere and Newton's gravitation.

2. The impossibility to achieve a universal symmetry for Einstein's gravitation caused by the curvature of space, as studied in Section 3, with consequential adoption of "covariance" and ensuing lack of prediction of the same numerical values under the same conditions at different times.

3. The incompatibility of Einstein's gravitation with 20th century doctrines, including special relativity, electrodynamics, and quantum mechanics, which is also caused by the curvature of space.

The return of gravitation to a flat space via its formulation in the Minkowski-Santilli isospace and isogeometry presented in paper [54] under the name of isogravitation, has permitted the achievement of the universal invariance for all gravitational line elements [27], with a direct and unequivocal compatibility of isogravitation with 20th century theories.

The aspect of this new vista in gravitation important for this paper is the transition from the failed "unification" of the gravitational and electromagnetic fields, to their "identification" with ensuing possibility of the laboratory creation of the gravitational field according to proposals dating back to 1974 [66].

In turn, the capability to create an attractive gravitational field, combined with the predicted gravitational repulsion between matter and antimatter, is expected to initiate the study of fundamentally new propulsions beyond the rather primitive Newtonian propulsion of current use nowadays that are known as isogeometric propulsion [55].

\subsection{Interstellar Travel}

\subsubsection{Foreword}

As it is well known, the initiation of scientific studies on interstellar travel has been opposed by the orthodox physics community via discreditation and other means because interstellar travel requires arbitrary speeds that are popularly believed not to be permitted by Einstein's theories. 
This posture is essentially based on the belief of the universal validity of Einstein's theories for all possible conditions existing in the large and small scale structure of the universe, expectedly, until the end of time.

In reality, it is the fate of all theories to have precise conditions of exact applicability beyond which the theories are only approximately valid or totally inapplicable. In the author's view, this is precisely the case of interstellar travel.

In this subsection we shall outline our current mathematical knowledge of interstellar travel, with a warning that its serious understanding requires a technical knowledge of our most advanced known mathematics for matter and antimatter, known as hypermathematics [50].

\subsubsection{Inapplicability of Einstein's Theories for Interstellar Travel}

Interstellar travel cannot be achieved in the event the necessary fuel has to be carried along in tanks. Consequently, in the author's view, interstellar travel can only be achieved by continuously extracting the necessary energy from space conceived as a universal substratum with a very large energy density, also known as zero point energy [72].

The process of energy extraction from space renders interstellar travel a strictly interior dynamical problem irreversible over time for which Einstein's theories cannot be minimally formulated in a consistent form due to the need for its Lie-admissible formulation for quantitative treatments.

In addition, we expect the need of the antimatter-type energy, known as isodual energy, and other aspects require the joint treatment of matter and antimatter which can be best done via of the most advanced mathematics known by mankind, the hypermathematics [50].

It should be indicated that, rather than being far fetched as popularly believed, the extraction of energy from space has resulted to be necessary for a consistent representation of the synthesis of the neutron from the hydrogen via a longitudinal impulse, called the etherino which is itself superluminal [58].

\subsubsection{The Universal Substratum for Particles and \\ Electromagnetic Waves}

The first scientific work written by the author [73] (in 1957 when attending high school) dealt with the resolution of the historical problem of the "ethereal wind," namely, the expectation that, in the event space has physical characteristics similar to medium, the orbiting of Earth around the Sun would be slowed down due to the resistance caused by said medium, which is contrary to evidence.

The argument $t$ of paper [73] is that electromagnetic waves as well as the elementary particles composing matter are "pure oscillations" of the universal substratum in the sense that the oscillations occur at specific points of space without any oscillating "little mass."

This is evidently the case for electromagnetic "waves" that, in the author opinion, cannot exist without a medium, but we have a similar occurrence also for the structure of the electron which is known to be a "pure oscillation" of a point of space with a frequency of $0.829 \times 10^{10} \mathrm{~Hz}$ without any little mass" oscillating in its interior.
Consequently, the main point of Ref., [73] is that, when we move an object, we have no solid substance at all because we merely have the propagation through space of the oscillations characterizing the object without any possible "ethereal wind."

Paper 73 then concluded that space should have characteristics similar to "rigidity" due to the transverse character of electromagnetic waves that prevent other structures of space, such as that as a "fluid" for which transverse waves cannot notoriously exist. The very high value of the speed of electromagnetic waves then suggested for space to have an extremely high energy density.

A central conception underlying the view here presented is therefore that space is totally full and matter is totally empty. This conception, which is clearly against our sensory perception, can be visualized by assuming the capability of "seeing" an electron. Under our ordinary time the electron appears as solid sphere due to the high value of the frequency of the oscillations. However, in the event said frequency goes to zero the electron would disappear. The same holds for the other elementary particles and, therefore, for all matter.

It should be noted that the conception of matter as being "pure oscillations" of space seems to be needed for other features of interstellar travel indicated below. It should be also noted that, according to a widely adopted view (see, e.g., Ref. [72]), matter is conceived as being "immersed" in the universal substratum.

\subsubsection{The Universal Substratum for Matter and Antimatter}

The above conception implies that the energy contained in space can be essentially conceived to be similar to energies in our environment, such as the potential energy, thus being a positive energy $E$ measured with the conventional positive units, such as erg. This view is confirmed in particle laboratories since we can extract from space photons and/or particles via the use of conventional energies.

In the author's view, the above conception of space is basically insufficient to achieve interstellar travel due to the need for space to be the universal substratum also for antimatter photons and antimatter particles [74].

In the latter case, a number of consistency conditions require that the antimatter substratum should have negative energy, called isodual energy indicated as $E^{d}$ although referred to negative units, known as isodual units indicated $\operatorname{erg}^{d}$, where the upper symbol denotes the conjugation from matter to antimatter called isoduality and given by an anti-= Hermitean map.

Additional requirements of compatibility with our current knowledge of matter and antimatter (such as the capability of extracting from space both, particles and antiparticles) imply that space is a superposition of equal amounts conventional and isodual energy, each with extremely high density, which can be best represented as a hyperstructure [75].

\subsubsection{Hyperspeeds}

A main assumption of the propulsion for interstellar travel, here [presented is the capability by a "matter-spaceship" to extract from space antimatter/isodual energy [74] because 
matter and antimatter are predicted to experience gravitational repulsion at all levels of study from Newtonian mechanics to second quantization [55].

In fact, the author has not been able to achieve a mathematical propulsion model when a matter-spaceship extract conventional energy from space, since conventional (positive) energy is the origin of gravitational attraction according to Einstein.

By contrast, in the event a matter-spaceship is capable of extracting antimatter-energy from space propulsion in all directions opposite said extraction become possible at arbitrary speeds evidently proportional to the extracted antimatter energy.

It should be noted that this view is not generally adopted in current studies on interstellar travel, since they assume the achievement of propulsion via the extraction form space of positive energy from a matter-spaceship [72].

At this point, the mathematical, treatment of the propulsion here suggested under the name of hypergeometric propulsion becomes rather complex (see Chapter 4 of monograph [55] for technical details of the sublease of isogeometric propulsion, and the various references on antimatter quoted in this paper).

The main assumption of the proposed hypergeometric propulsion is that the spaceship 'achieves propulsion buy changing the geometry in its environment without any motion being perceived by its operators, thus allowing instantaneous acceleration, singular trajectories, lack of sonic boom when traveling in atmosphere at supersonic speeds and other anomalies. Hypermathematics is needed for the joint use of matter ands antimatter energies.

The simpler case of the isogeometric propulsion studied in details in Ref.[55] can be mathematically represented via the invariant ;lifting of the spacetime line element of special relativity

$$
\begin{gathered}
\Delta x^{2}+\Delta y^{2}+\Delta z^{2}-c^{2} \Delta t^{2} \equiv \\
\left.\equiv \Delta x^{\prime 2} \hat{I}_{x}+\Delta y^{\prime 2} \hat{I}_{y}+\Delta z^{\prime 2}\right] \operatorname{hat}_{z}-c^{2} \Delta t^{\prime 2} \hat{I}_{t} \equiv
\end{gathered}
$$

where

$$
\Delta x=x_{1}-x_{2}, \Delta y=y_{1}-Y_{2}, \Delta z=z_{1}, \Delta t=-t_{r} 1-t_{2}
$$

represents the motion in space and time.as seen by an outside observer.

Isogeometric propulsion (for a spaceship composed of matter )occurs via the extraction from space of antimatter energy in a given direction, say $\Delta x$, resulting in a propulsion in the direction $-\Delta x$ opposite that of said extraction due to the repulsion of matter and antimatter.

The extraction of antimatter energy, say, in the $x=$ direction, is mathematically represented via the transition from the conventional unit 1 of our spacetime to the isounit in the indicated direction, $\hat{I}_{x}$ with consequential decrease of the distance $\Delta x^{\prime}$ in the indicated direction due to the invariance $\Delta x=\Delta x^{\prime} \hat{I}_{x}$.

Mathematically, there is no limit in the acquired speeds, which can be millions of times the speed of light $c$, since the isounit in the $x$-direction can be extremely large in which cased, the actual distance $\Delta x^{\prime}$ covered by the spaceship is infinitesimally small.

Some of the consequences of the above isogeometric locomotion can be expressed as follow:

A. The extraction of any energy from space causes a deformation of the stricture of space, called mutation.

B. The size of the spaceship perceived by external and internal observers can be dramatically different.

C.There cannot be mutation of space without a corresponding mutation of time, thus illustrating the name of ßpacetime machine" in Ref. [55].

D. The visual detection of a spaceship by an external observer does not mean that the spaceship exists at his/her time.

E. The lapse of time measured by the spaceship operator can be much smaller than the lapse of time perceived by an external observer.

The generalization of the above isogeometric propulsion to the hypergeometric form is excessively complex for the limited scope of this presentation and will be presented elsewhere.

\subsubsection{Hypercommunications}

At interstellar distances, electromagnetic communications are like the smoke signals of the American Indians because they are excessively slow compared to the distances at hand. Since interstellar travel cannot even be conceived without effective communications, we find again the need of surpassing the speed of light which has been opposed for one century by the orthodox physics community.

Yet, there already exist in the refereed literature experiments showing the propagation of signals faster than light (see, e.g., Ref. [19]); signals propagating at a large multiple the speed of light have already been computed for the longitudinal propagation of energy in the synthesis of the neutron [58]; and, according to (unpublished) initial calculations, the Lie-Isotopic and Lie-admissible coverings of Maxwell's equations predict virtually instantaneous longitudinal signals particularly if space is assumed to have a characteristic similar to external high "rigidity."

\subsubsection{Hyperfading}

Trajectory corrections to avoid collisions can indeed be done for spaceships traveling at interplanetary speeds, but said corrections cannot be done when traveling at interstellar speeds that are expected to be millions of time the speed of light.

A conceivable solution, here suggested by the author under the name of hyperfading, is the capability of interstellar spaceships to alter their physical structure in such a wave to cross through astrophysical bodies without collisions or damage. This also implies the capability to enter oceans without creating any wave.

Rather than being far fetched, this possibility is within current mathematical possibilities. In fact, the mutation of space which is inherent in the hypergeometric locomotion implies the alteration of the spacetime of the spaceship and of its inhabitants. Hyperfading is then mathematically possible 
via the control of time permitted by antimatter, a conceivable dramatic reduction of the characteristic frequencies of the spaceship and its inhabitants under which the spaceship literally "fades away" from the universe, and other means.

Thanks to advances in antimatter with the ensuing isogeometric propulsion [55] in which hyperfading could be achieved due to the structural alteration of the local spacetime, the control of time predicted from a suitable use ofisodual energies (negative energies referred to negative units), and other means.

It is hoped that young minds of any age will ignore biased obstructions from the orthodox physics community and initiate indeed scientific studies on the fascinating open problems connected to interstellar travel because, in the author's view, the achievement by mankind of interstellar travel is only a question of time.

\section{Concluding Remarks}

During the studies on axiom-preserving isotopies and genotopies of 20th century theories, the author has stated various times that:

Rather than abusing the names of Lorentz, Poincaré, Einstein and other founders of 20th century physics by applying their theories under interior dynamical conditions they were not intended for, and cannot be properly formulated and tested, the best way to honor their names is to maintain their axioms, and enlarge their conditions of exact applicability via the use of broader mathematics specifically built for interior conditions.

The implications in surpassing 20th century theories for interior dynamical conditions are so deep for all quantitative sciences to imply a "new scientific era," as indicated in the tile of Ref. [42].

\section{References}

[1] L. V. Lorentz, Philos. Mag. 34, 287 (1867)

[2] H. Poincaré, Archives Nerland. des Sciences Exactes et Naturelles, 2, vol. 5, 252-278 (1900).

[3] A. Einstein, Ann. Phys. (Leipzig) 17, 891 (1905).

[4] E. Hubble, Proceedings of the National Academy of Sciences of the United States of America,, 168 (1929).

[5] F. Zwicky, Proceedings of the National Academy of Sciences of the United States of America, 773 (1929).

[6] M. J. Rees, Nature, 468 (1966).

[7] A. B. Whiting, "The expansion of space: free-particle motion and the cosmological redshift," The Observatory, 174 (2004) http://adsabs.harvard.edu/abs/2004Obs...124174W

[8] P. Riess et al. Astronomical Journal, 1009 [1998); S. Perlmutter et al. Astrophysical Journal, 565 (1999).

[9] R. M. Santilli, "Embedding of Lie algebras in Lie-admissible structures," Nuovo Cimento, 570 (1967), available in free download from the link http://www.santilli-foundation.org/docs/Santilli-54.pdf
[10] R. M. Santilli, "Can strong interactions accelerate particles faster than the speed of light?" Lettere Nuovo Cimento, 145 (1982) www.santilli-foundation.org/docs/Santilli-102.pdf

[11] R. M. Santilli, "Isonumbers and genonumbers of dimension 1, 2, 4,8 , their isoduals and pseudoduals, and hidden numbers of dimension 3, 5, 6, 7, Ó Algebras, Groups and Geometries Vol. 10, 273-321 http://www.santilli-foundation.org/docs/Santilli-34.pdf

(1993)

[12] R. M. Santilli, "Nonlocal-Integral Isotopies of Differential Calculus, Mechanics and Geometries," in Isotopies of Contemporary Mathematical Structures, P. Vetro Editor, Rendiconti Circolo Matematico Palermo, Suppl. Vol. 42, 7-82 (1996), http://www.santilli-foundation.org/docs/Santilli-37.pdf

[13] R. M. Santilli, "Lie-admissible invariant representation of irreversibility for matter and antimatter at the classical and operator levels," Nuovo Cimento B bf 121, 443 (2006), available in free pdf download from the link http://www.santilli-foundation.org/docs/Lie-admiss-NCB-I.pdf

[14] J. V. Kadeisvili, “The Rutherford-Santilli Neutron," Hadronic J. 2008; 31， 1, http://www.i-b-r.org/Rutherford-Santilli-II.pdf available in $\mathrm{html}$ version in the website http://www.i-b-r.org/Rutherford-Santilli-neutron.htm

[15] R. M. Santilli, Foundation of Theoretical Mechanics, Volume I (1978) [15a], and Volume II (1982) [15b], Springer-Verlag, Heidelberg,

http://www.santilli-foundation.org/docs/Santilli-209.pdf $\mathrm{http} / / / \mathrm{www}$.santilli-foundation.org/docs/santilli-69.pdf

[16] R. M. Santilli, Hadronic Mathematics, Mechanics and Chemistry, Volumes I [16a], II [16b], III [16c], IV [16d] and V [16e] International Academic Press 2008, http://www.i-b-r.org/Hadronic-Mechanics.htm

[17] E. Wall, The Physics of Tachions, Hadronic Press (1995).

[18] A. Enders and G. Nimtz, J- Phys.I (France), 1693-1698 (1992)

[19] G. Nimtz, Foundations in Physics,, 1193-1199 (2011)

[20] D. Sokolovski and E. Akhmatskaya, Phys. Rerv. A, 052110 (2013).

[21] A. Ereditato et al., "Measurement of the neutrino velocity with the OPERA detector in the CNGS beam," CERN preprint, http://www.santilli-foundation.org/docs/OPERA-experiment.pdf

[22] N. Yu et al., "Measurement of the Velocity of Neutrinos from the CNGS Beam with the Large Volume Detector," Phys. Rev. Lett. v.109, 070801(2012) [70d]

[23] R. M. Santilli, "Lie-isotopic Lifting of Special Relativity for Extended Deformable Particles," Lettere Nuovo Cimento, 545 (1983), http://www.santilli-foundation.org/docs/Santilli-50.pdf

[24] R. M. Santilli, "Lie-isotopic Lifting of Unitary Symmetries and of Wigner's Theorem for Extended and Deformable Particles," Lettere Nuovo Cimento Vol. 38, 509 (1983), http://www.santilli-foundation.org/docs/Santilli-51.pdf

[25] R. M. Santilli, "Isotopies of Lie Symmetries," I (basic theory) and II (isotopies of the rotational symmetry), Hadronic J. Vol. 8, 36 and 85 (1985), http://www.santilli-foundation.org/docs/santilli-65.pdf

[26] R. M. Santilli, "Isotopic Lifting of the SU(2) Symmetry with Applications to Nuclear Physics," JINR rapid Comm. Vol. 6. 24-38 (1993), http://www.santilli-foundation.org/docs/Santilli-19.pdf. 
[27] R. M. Santilli, "Nonlinear, Nonlocal and Noncanonical Isotopies of the Poincare' Symmetry," Moscow Phys. Soc. Vol. 3, $255 \quad$ (1993), http://www.santilli-foundation.org/docs/Santilli-40.pdf

[28] R. M. Santilli, "Recent theoretical and experimental evidence on the synthesis of the neutron," Communication of the JINR, Dubna, Russia, No. E4-93-252 (1993), published in the Chinese J. System Eng. and Electr. Vol. 6, 177 (1995), http://www.santilli-foundation.org/docs/Santilli-18.pdf

[29] R. M. Santilli, "Isorepresentation of the Lie-isotopic SU(2) Algebra with Application to Nuclear Physics and Local Realism," Acta Applicandae Mathematicae Vol. 50, 177 (1998), $\mathrm{http} / / / \mathrm{www}$. santilli-foundation.org/docs/Santilli-27.pdf

[30] R. M. Santilli, "Isominkowskian Geometry for the Gravitational Treatment of Matter and its Isodual for Antimatter," Intern. J. Modern Phys. D, 351 (1998), http://www.santilli-foundation.org/docs/Santilli-35.pdf

[31] J. V. Kadeisvili, "Direct universality of the Lorentz-Poincare'-Santilli isosymmetry for extended-deformable particles, arbitrary speeds of light and all possible space-times" in Photons: Old problems in Light of New Ideas, V. V. Dvoeglazov Editor Nova Science (2000, available as free download from http://www.santilli-foundation.org/docs/Santilli-25.pdf

[32] A. K. Aringazin and K. M. Aringazin, Üniversality of Santilli's iso-Minkowskian geometry" in Frontiers of Fundamental Physics, M. Barone and F. Selleri, Editors Plenum 91995), available as free download from http://www.santilli-foundation.org/docs/Santilli-29.pdf

[33] R. M. Santilli, Isotopic Generalizations of Galilei and Einstein Relativities, Vols. I [14a] and II [14b] (1991), International Academic

http://www.santilli-foundation.org/docs/Santilli-01.pdf http://www.santilli-foundation.org/docs/Santilli-61.pdf

[34] R. M. Santilli, Elements of Hadronic Mechanics, Vol. I (1995) [15a], Vol. II 91995) [15b] Academy of Sciences, Kiev, http://www.santilli-foundation.org/docs/Santilli-300.pdf http://www.santilli-foundation.org/docs/Santilli-301.pdf

[35] A. K. Aringazin, A. Jannussis, F. Lopez, M. Nishioka and B. Veljanosky, Santilli's Lie-Isotopic Generalization of Galilei and Einstein Relativities, Kostakaris Publishers, Athens, Greece

(1991), http://www.santilli-foundation.org/docs/Santilli-108.pdf

[36] J. Lôhmus, E. Paal, and L. Sorgsepp, Nonassociative Algebras in Physics Hadronic Press, Palm Harbor, Florida (1994), http://www.santilli-foundation.org/docs/Lohmus.pdf

[37] D. S. Sourlas and G. T. Tsagas, Mathematical Foundation of the Lie-Santilli Theory, Ukraine Academy of Sciences (1993), http://www.santilli-foundation.org/docs/santilli-70.pdf

[38] R. M. Santilli, "Nonlocal-Integral Isotopies of Differential Calculus, Mechanics and Geometries," in Isotopies of Contemporary Mathematical Structures, P. Vetro Editor, Rendiconti Circolo Matematico Palermo, Suppl. Vol. 42, 7-82 (1996), http://www.santilli-foundation.org/docs/Santilli-37.pdf

[39] J. V. Kadeisvili, Santilli's Isotopies of Contemporary Algebras, Geometries and Relativities, Ukraine Academy of Sciences, Second edition

(1997), http://www.santilli-foundation.org/docs/Santilli-60.pdf.
[40] Raul M. Falcon Ganfornina and Juan Nunez Valdes, Fundamentos de la Isoteoria de Lie-Santilli, International Academic Press (2001), http://www.i-b-r.org/docs/spanish.pdf

[41] A. K. Aringazin, "Studies on the lie-Santilli IsoTheory with Unit of general Form," Algebras, Groups and Geometries, 299 (2011),

http://www.santilli-foundation.org/docs/Aringazin-2012.pdf

[42] I. Gandzha and J. Kadeisvili, New Sciences for a New Era: The Mathematical, Physical and Chemical Discoveries of Ruggero Maria Santilli, Sankata Printing Press, Nepal (2011), http://www.santilli-foundation.org/santilli-scientific-discoverie s.html

[43] R. M. Santilli, "Experimental Verifications of IsoRedShift with Possible Absence of Universe Expansion, Big Bang, Dark Matter, and Dark Energy," The Open Astronomy Journal, 124 (2010),

http://www.santilli-foundation.org/docs/Santilli-isoredshift.pdf

[44] R. M. Santilli, G. Amato and G. West, "Experimental Confirmation of the IsoRedShift at Sun at Sunset and Sunrise with Consequential Absence of Universe Expansion and Related Conjectures," Journal of Computational Methods in Sciences and Engineering, Vol. 12, pages 165-188 (2012). http://www.santilli-foundation.org/docs/Confirmation-sun-IRS .pdf

[45] H. Ahmar, G. Amato, J. V. Kadeisvili, J. Manuel, G. West, and O. Zogorodnia, "Additional experimental confirmations of Santilli IsoRedShift and the consequential absence of the universe expansion," Journal of Computational Methods in Science and Engineering, 321 (2013), http://www.santilli-foundation.org/docs/IRS-confirmations-21 2.pdf

[46] R. M. Santilli, "Isominkowskian representation of cosmological redshifts and the internal redshift/blueshift of quasars," contributed paper in Frontiers of Fundamental Physics, M. Barone and F. Selleri, Editors, Plenum Press (1994) http://www.santilli-foundation.org/docs/ire-obd-1994.pdf

[47] R. M. Santilli,,"Representation of galactic dynamics via isoshifts without universe expansion, dark matter and dark energy," American Journal of Modern Physics Vol. 4, pages 26-43

(2015) http://www.thunder-energies.com/docs/dark-matter-2015.pdf

[48] R. Folman and E. Recami, "On the phenomenology of tachyon radiation", Found. Phys. Lett.,, , 2 (1995).

[49] E. Recami, M. Zamboni-Rached, C. A. Dartora, "Localized $\mathrm{X}$-shaped field generated by a superluminal electric charge," Phys. Rev. E, 027602 (2004).

[50] R. Anderson, "Outline of Hadronic Mathematics, Mechanics and Chemistry as Conceived by R. M. Santilli," American Journal of Modern Physics Vol. 4(5), pages 1-16 (2015) http://www.santilli-foundation.org/docs/hadronic-math-mec-c hem.pdf

[51] R. M. Santilli. Isotopic Generalization of Galileo and Einstein Relativities. Hadronic Press 1991, Volumes I [3a] and II [3b], http://www.santilli-foundation.org/docs/Santilli-01.pdf http://www.santilli-foundation.org/docs/Santilli-35.pdf

[52] R. M. Santilli, "Isorelativity," invited plenary lectures (2014) http://www.world-lecture-series.org/isorelativity-2014-i http://www.world-lecture-series.org/isorelativity-2014-ii http://www.world-lecture-series.org/isorelativity-2014-iii. 
[53] R. M. Santilli, "Isorelativity for matter and its isodual for antimatter," invited paper, American Journal of Modern Physics, kin press (2015)

[54] R. M. Santilli, "Rudiments of isogravitation for matter and its isodual for antimatter," American Journal of Modern Physics Vol. 4(5), pages 59-75 (2015) http://www.santilli-foundation.org/docs/isogravitation.pdf

[55] R. M. Santilli, Isodual Theory of Antimatter with Applications to Antigravity, Grand Unifications and Cosmology, Springer (2006).

[56] R. M. Santilli, "An intriguing legacy of Einstein, Fermi, Jordan and others: The possible invalidation of quark conjectures," Found. Phys. Vol. 11, 384-472 (1981) http://www.santilli-foundation.org/docs/Santilli-36.pdf

[57] Yu. Arestov, R. M. Santilli and V. Solovianov, "Experimental evidence on the isominkowskian character of the hadronic structure," Foundation of Physics Letters Vol. 11, pages 483-492

http://www.santilli-foundation.org/docs/Santilli-52.pdf

(1998)

[58] R. M. Santilli, "The etherino and/or the neutrino hypothesis," Foundation of Physics, 670 (2007). http://www.santilli-foundation.org/docs/EtherinoFoundPhys.p df

[59] R. M. Santilli, "A quantitative isotopic representation of the deuteron magnetic moment," in Proceedings of the International Symposium 'Dubna Deuteron-93, Joint Institute for Nuclear Research, Dubna, Russia (1994), http://www.santilli-foundation.org/docs/Santilli-134.pdf $<$ br $>$

[60] R. M. Santilli, "Nuclear realization of hadronic mechanics and the exact representation of nuclear magnetic moments," Intern. J. of Phys. Vol. 4, 1-70 (1998) http://www.santilli-foundation.org/docs/Santilli-07.pdf $<$ br $>$

[61] A. A. Bhalekar and R. M. Santilli, "Exact and invariant representation of nuclear magnetic moments and spins according to hadronic mechanics,": invited paper, to appear (2015).

[62] Thunder Energies Corporation, websiote, http://www.thunder-energes.com

[63] R. M. Santilli, Foundations of Hadronic Chemistry, with Applications to New Clean Energies and Fuels, Kluwer Academic Publishers (2001), http: //www.santilli-foundation.org/docs/Santilli-113.pdf Russian translation

http://i-b-r.org/docs/Santilli-Hadronic-Chemistry.pdf
[64] C. P. Pandhurnekar,"Advances on Alternative Fuels with Santilli Magnecular Structure," International Journal of Alternative Fuels, ISSN: 2051-5987, Vol.17, 2014, http://www.santilli-foundation.org/docs/Magnegas-2015.pdf

[65] R. M. Santilli, Isotopic, Genotopic and Hyperstructural Methods in Theoretical Biology, Ukraine Academy of Sciences, Kiev http://www.santilli-foundation.org/docs/santilli-67.pdf

[66] R. M. Santilli, "Partons and Gravitation: some Puzzling Questions," (MIT) Annals of Physics, Vol. 83, 108-157 (1974), http://www.santilli-foundation.org/docs/Santilli-14.pdf.

[67] O. M. P. Bilanik, V. K. Deshpande, and E. VC. G. Sudareshan, "Meta" Relativity, Americamn Journal of Physics, Vol. 30, pagez 718-723 (1962), http://www.santilli-foundation.org/docs/1962-006.pdf.

[68] A. O. Barut, Electrodynamics and Classical Theory of Fields and Particles (Dover, Nova Iorque, 1964), Classics of Science and Mathematics.

[69] T. G. Pavlopoulos, "Are we observing Lorentz viollagion in gamma rays bursts?, Physics letters B, 625, 13-18 (2005), http://www.santilli-foundation.org/docs/Extended-SR.pdf

[70] George P. Shpenkov, "On the Superluminal Speed in View of the Dialectical Model of the Universe," Infinite Energy, Vol. 77, pages 1-6, (2008); http://www.santilli-foundation.org/docs/Shpenkov.pdf

[71] D. M Bilaniuk and E. C. O. Sudarshan, "Particles beyond the light barrier," Physics Today, May 1969 http://www.santilli-foundation.org/docs/Sudarshan.pdf

[72] Mark McCandlish, Zero Point, Documentary https://vimeo.com/133170463

[73] R. M. Santilli, "Why space is rigid," (in Italian)Il Pungolo Verde, http://www.santilli-foundation.org/docs/Santilli-49.pdf

[74] R. M. Santilli, "Antigravity, Hadronic Journal, 257-284 (1994) http://www.santilli-foundation.org/docs/Santilli-13.pdf

[75] B. Davvaz, R. M. Santilli and T. Vougiouklis, Studies Of Multi-Valued Hyperstructures For The characterization Of Matter-Antimatter Systems And Their Extension Proceedings of the Third International Conference on the Lie-Admissible Treatment of Irreversible Processes, C. Corda, Editor, Kathmandu University (2011), pages 45-57 http://www.santilli-foundation.org/docs/Davvaz-Sant-Vou.pdf. 